\title{
Differential Adverse Events Between TNF- $\alpha$ Inhibitors and IL-17 Axis Inhibitors for the Treatment of Spondyloarthritis
}

Maria Antonelli, $M D^{1}$

Muhammad A. Khan, $M D^{1}$

Marina N. Magrey, $M D^{2, *}$

\author{
Address \\ ${ }^{1}$ Case Western Reserve University/Metrohealth Medical Center, Cleveland, $\mathrm{OH}$, USA \\ ${ }^{*}, 2$ Division of Rheumatology, Department of Medicine, Case Western Reserve \\ University/Metrohealth Medical Center, Cleveland, $\mathrm{OH}$, USA \\ Email: mmagrey@metrohealth.org
}

Published online: 19 April 2015

(C) Springer International Publishing AG 2015

This article is part of the Topical Collection on Spondyloarthritis and Psoriatic Arthritis

Keywords Spondyloarthritis · Adverse events · TNF- $\alpha$ inhibitors · IL-17 • Malignancy · Infections

\section{Opinion statement}

Availability of biologics, particularly tumor necrosis factor alpha (TNF- $\alpha$ ) inhibitors, has revolutionized the treatment of spondyloarthritis $(\mathrm{SpA})$. The main side effect associated with TNF- $\alpha$ inhibitors is increased rate of infection. Despite significant concerns about tolerability and adverse events of TNF- $\alpha$ inhibitors in treatment of SpA, they have stood the test of time with acceptable safety outcomes. However, there is a subset of patients with psoriatic arthritis (PsA) and ankylosing spondylitis (AS) who fail to respond to TNF- $\alpha$ inhibitors, lose efficacy over a period of time, or develop serious adverse events, particularly opportunistic infections. Newer therapeutic options have become available for these patients including interleukin-17 (IL-17) axis antagonists. Their safety data is limited to clinical trials only, with no registry data available as yet. There are no large head-to-head comparative trials between TNF- $\alpha$ inhibitors and IL-17 axis inhibitors. Based on data from clinical trials of relatively limited duration, infection rates are quite similar between these two classes of biologics but there are, as yet, no reports of reactivation of opportunistic infections like tuberculosis with IL-17 axis antagonists. However, pre-screening for tuberculosis and prophylaxis in appropriate candidates is still needed. The current available data have shown no other major discrepancies in the adverse events between TNF- $\alpha$ inhibitors and IL-17 axis 
inhibitors. More data is needed to effectively determine the comparative safety of TNF- $\alpha$ inhibitors versus IL-17 axis antagonists.

\section{Introduction}

Spondyloarthritis (SpA) is a heterogeneous group of conditions composed of inflammatory axial and peripheral arthritis, uveitis, inflammatory bowel disease, psoriasis, dactylitis, and enthesitis [1]. Psoriatic arthritis affects $0.1-1.0 \%$ of the general population $[2,3]$, while the prevalence of ankylosing spondylitis is likely very close to $1 \%$ [3]. SpA was previously only treated with non-steroidal anti-inflammatory drugs (NSAIDs) and traditional disease-modifying anti-rheumatic drugs (DMARDs), with varying success. The advent of TNF inhibitors heralded enormous success with treatment of both radiographic and non-radiographic spondylitis. Availability of novel IL-17 axis inhibitors has opened more treatment options for these diseases. The focus of this review will be on the differential adverse events among the TNF- $\alpha$-inhibiting and IL-17-modulating classes of agents.

\section{TNF-a Inhibitors}

Pro-inflammatory cytokines such as tumor TNF- $\alpha$ play a central role in the inflammation underlying SpA, and agents that selectively block TNF- $\alpha$ have proved highly effective in treating PsA and ankylosing spondylitis (AS). They work by blocking the cytokine itself (infliximab, adalimumab, and golimumab) or its antigen-binding fragment ( $\left.\mathrm{Fab}^{\prime}\right)$ (certolizumab), or its receptor (etanercept), and prevent the activation and expansion of T cells, leading to a decrease in overall inflammation.

Since TNF- $\alpha$ is a pro-immune cytokine involved in antimicrobial type 1 immunity, patients treated with TNF- $\alpha$ antagonists have increased risk of infections $[4 \bullet, 5,6]$, and a large majority of these are upper respiratory tract infections [7•]. An increased risk of opportunistic infections, particularly fungal infections and tuberculosis, is noted as a black box warning with these agents. In addition, rates of demyelinating disorders, lymphomas, and solid tumors have been noted to be increased in populations using these medications [6]. Availability of TNF- $\alpha$ inhibitors on the commercial market for a prolonged period of time has provided information about long-term safety from post-marketing surveillance, registries, long-term follow-up studies, and voluntary reporting $[4 \bullet, 7 \bullet \bullet, 8-10]$. Table 1 outlines side effects, malignancies, opportunistic infections, and deaths for each drug included in this review.

The most common ( $>5 \%$ in study patients) non-serious adverse event (AE) for all TNF- $\alpha$ antagonists is infections. After nearly 3 years of follow-up, the most common AE in the adalimumab trial in PsA patients were similar to those in the first 6 months of the study: upper respiratory tract infections, nasopharyngitis, and sinusitis were seen in 21.5, 17.4, and $10.7 \%$, respectively [8]. Similar AE were seen in AS patients in the ATLAS trial over a period of 2 years [9]. A metaanalysis of randomized placebo-controlled trials of TNF- $\alpha$ inhibitors in patients with psoriasis and PsA patients indicates an odds ratio (OR) of 1.09 (95\% 


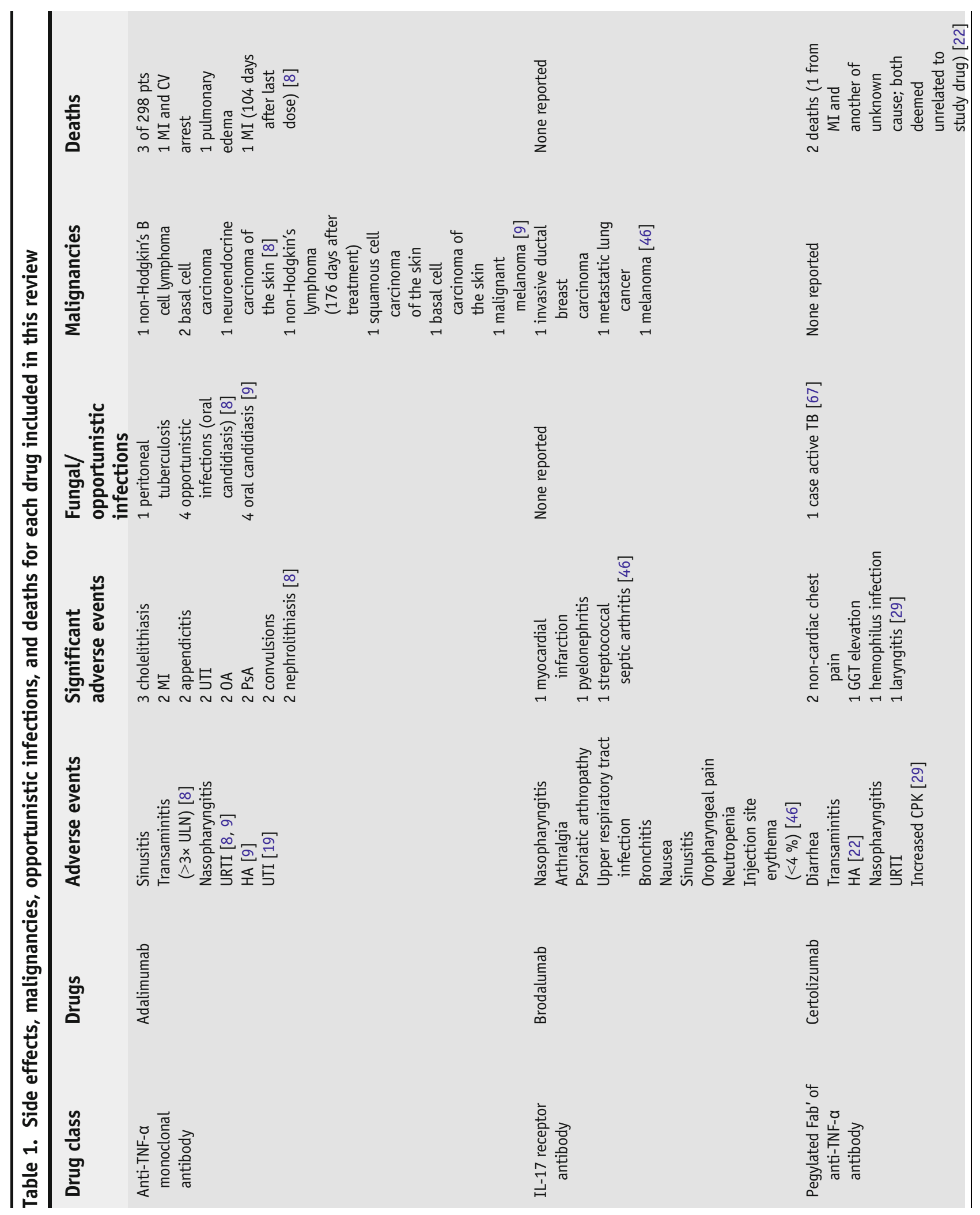




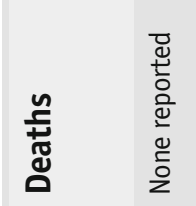

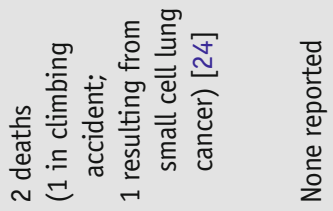

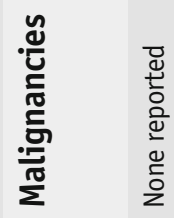

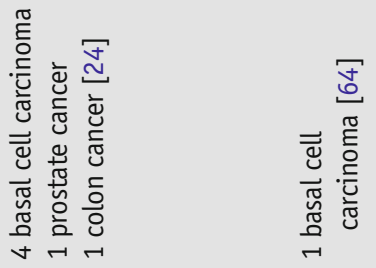

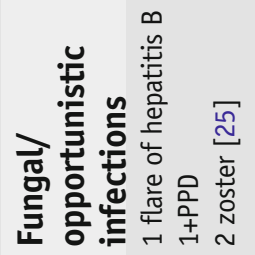

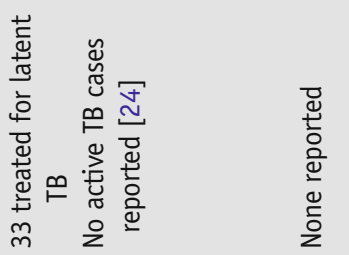

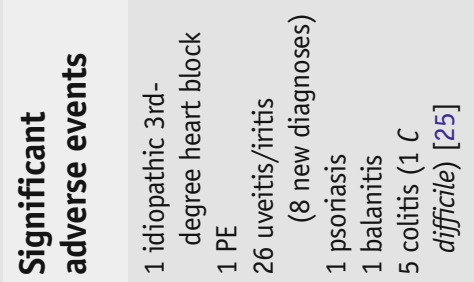

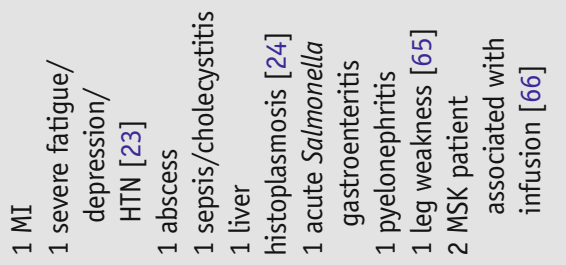

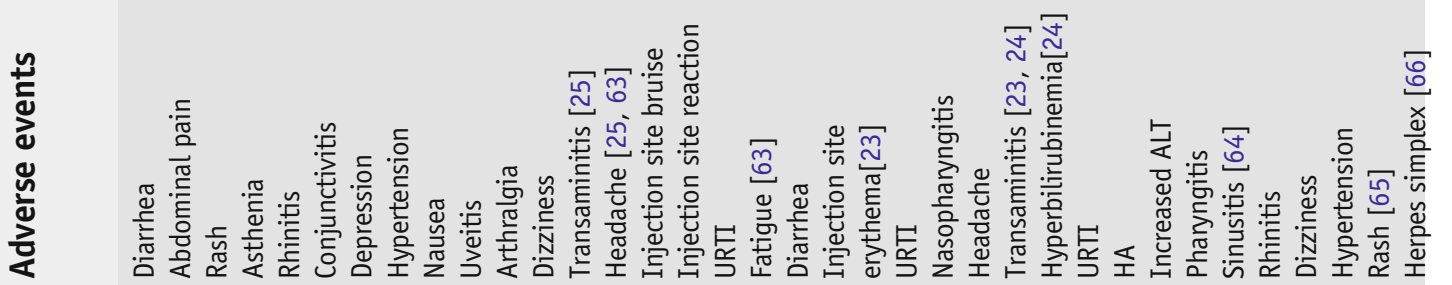

气ั

节

. ․ㅠ.

兽

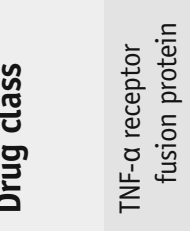
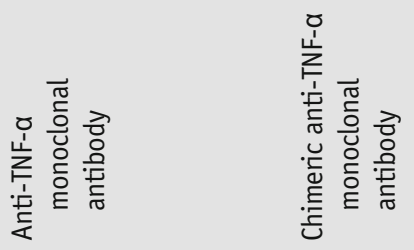


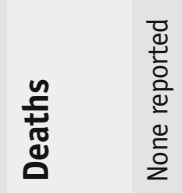

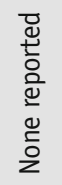

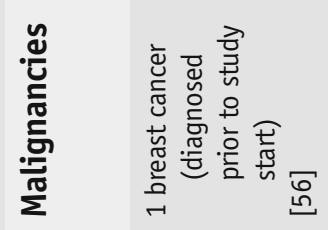

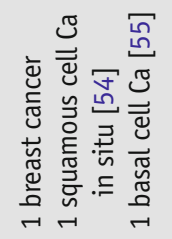

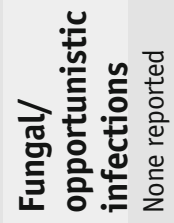

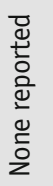

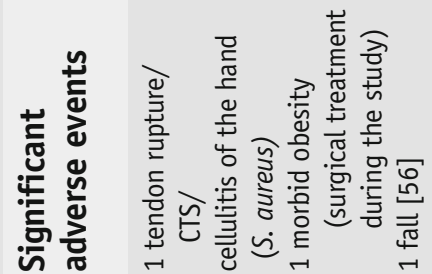

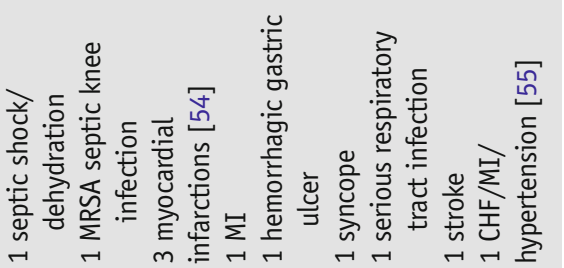

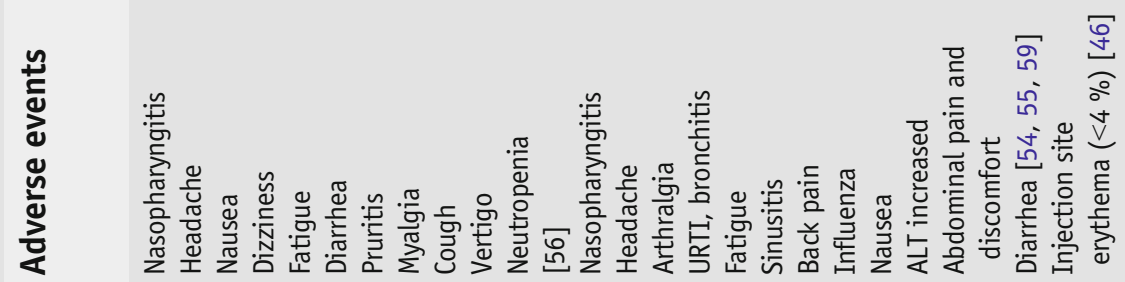

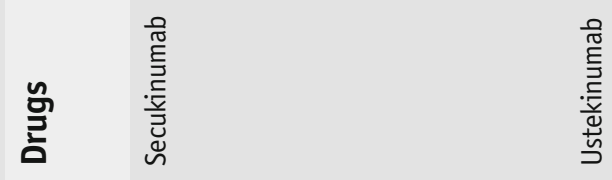

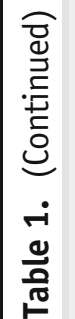

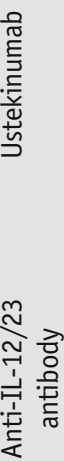


confidence interval (CI) $0.87,1.37)$ for any infectious event in patients treated with TNF inhibitors $[7 \bullet]$. Generally speaking, common infection rates are not dissimilar from among the different TNF-inhibitor trials. Reactivation of remote hepatitis B (HBV) infection has been seen with the use of TNF inhibitors [4•].

There is enormous data available about the use of TNF inhibitors in RA, and the findings of the longer-term data are generally consistent with those of shortterm trials. In the Italian GISEA registry which includes 2769 RA patients on long-term anti-TNF therapies, the overall incidence of serious infections was 31.8 events per 1000 patient-years of follow-up (95\% CI 25.2-38.3); this risk was numerically higher during the first year of treatment compared to the second year, but it was not statistically significant $(p=0.8)$ [10]. This incidence was similar to the prior German data from the RABBIT registry [11]. In the GISEA registry, multivariate analysis showed that steroid is a significant predictor of infection in TNF-inhibitor use, OR 1.633 (95\% CI 1.01-2.644) [10]. In univariate analysis, advanced age at initiation of TNF-inhibitor therapy is associated with risk of serious infection $(p<0.0001)$ [10].

\section{Tuberculosis and Opportunistic Infections}

TNF- $\alpha$ inhibitor therapy is associated with an increased risk of granulomatous infection, most notably reactivation of tuberculosis (TB). In latent tuberculosis infection (LTBI), few mycobacteria are housed in a granuloma; introduction of the TNF antagonist upsets this barrier and allows mycobacteria to reactivate and progress to active TB infection [12]. Prophylactic treatment has lowered this risk to reactivation [12].

Because studies of individual TNF inhibitors differ in details of study design, it has been difficult to discern differences in reactive TB rates among each discrete agent. However, it appears monoclonal antibodies to TNF carry a higher risk of reactivating latent TB. One US study demonstrated that infliximab has a higher rate of TB compared to etanercept (54 and 28 per 100,000 treated patients for infliximab and etanercept, respectively) [13]. Based on the French Research Axed on Tolerance of bIOtherapies (RATIO) registry, which includes patients with a variety of inflammatory and autoimmune diseases on biologic therapies, 69 cases of $\mathrm{TB}$ were reported over a total of 57,711 patient-years; these cases were related to infliximab and adalimumab use, but none of them had received the currently recommended chemo-prophylactic treatment for latent TB [14]. The British Society for Rheumatology Biologics Register (BSRBR) confirmed similar results in a cohort of 10,712 patients over median 3.2 years, finding a 3 - to 4 -found higher rate of TB in RA patients on infliximab and adalimumab compared to etanercept [15].

The RATIO registry also has found that infliximab and adalimumab carry a higher risk of a variety of serious non-tuberculous opportunistic infections compared to etanercept [16]. The same registry found a significantly higher risk of herpes zoster infection with monoclonal antibodies to TNF (adalimumab and infliximab) compared to the soluble TNF- $\alpha$ receptor, odds ratio (OR) 3.49 (95\% CI 1.12-10.90, $p=0.0316$ ) [17]. A similar trend was seen in the same registry regarding Legionella pneumophila infection; the standardized incidence ratio (SIR) for all TNF inhibitors was 13.1 compared to the general French population. 
However, the SIR for infliximab and adalimumab were higher, 15.3 and 31.8, respectively, in comparison to etanercept, SIR 2.4 [18].

As such, pre-screening for tuberculosis, prophylaxis in appropriate candidates as well as consideration of zoster vaccine, and continued surveillance remain imperative in SpA patients being considered for TNF-blocker therapy.

As TNF has been implicated in the pathogenesis of malignancy [6], there has been a concern for increased risk of malignancy with TNF- $\alpha$ inhibitor use in all autoimmune diseases. Including patients with all indications for the drug, the rate of lymphomas has been noted in adalimumab to be $0.1 / 100$ patient-years and the rate of non-melanomatous skin cancer to be $0.2 / 100$ patient-years [19•]. The French RATIO registry observed a discrepancy among agents; in a series of 38 cases of lymphoma in patients with a variety of autoimmune diseases on anti-TNF therapy, a significant risk was found to be related to infliximab or adalimumab use versus etanercept, OR 4.12 (95\% CI 1.3612.49) and OR 4.73 (95\% CI 1.27-17.65), respectively [20].

Unfortunately, this relationship remains unclear as conflicting data abound. In the psoriasis literature, it is reported that the risk of lymphoma as a result of TNF-antagonist therapy is approximately equivalent to the lifetime risk without TNF-blocker therapy $[4 \bullet]$. One long-term use report indicates that the observed number of malignancies in AS and PsA were similar to the number expected for age- and sex-matched populations [19•]. In a meta-analysis of TNF-blocker use in psoriasis and PsA patients, the OR for malignancy was not significant: 1.48 $(95 \%$ CI $0.71,3.09)[7 \bullet \bullet]$. In this meta-analysis of 20 trials which included studies of each of the five TNF antagonists, more than $70 \%$ of the 28 malig-

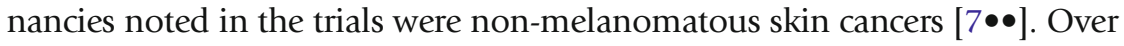
10 years, in one Belgian population, the incidence of malignancy after one or more anti-TNF therapies for SpA was noted to be 500.1 per 100,000 patient years, higher than the general population [21]. However, it is noted that this controversial relationship stems from the concern that some increased risk may be disease related and not treatment related.

Laboratory abnormalities including transaminitis, bilirubinemia, and mild creatinine kinase elevations (CPK) have been noted with TNF inhibitors. Transient elevation of liver enzymes was seen with adalimumab [8], certolizumab [22], and golimumab [23]. Similarly, in the golimumab study of PsA patients, Kavanaugh et al. noted hyperbilirubinemia in study patients [24]. In the AS trial of certolizumab, $5.1 \%$ of patients receiving the study agent had increased blood CPK. This was noted to be transient and resolved with continued therapy [24]. Cytopenias have been reported in TNF-inhibitor trials, including thrombocytopenia, lymphocytopenia, and neutropenia $[25,26]$. These cytopenias are generally mild and transient.

\section{Skin Reactions and Extra-Articular Features}

Injection site reactions have been noted in all TNF- $\alpha$ inhibitors. Rates of this reaction are seen more often in etanercept than in infliximab and adalimumab $[4 \bullet]$. Occurrence of a new-onset psoriasis, particularly palmo-plantar pustular 


\section{Inflammatory Bowel Disease}

psoriasis, has been noted $[4 \bullet]$. The cause of this is unclear, but treating topically, increasing the TNF-antagonist dose [4•], changing to another biologic agent [4•], or stopping the agent [27] has been noted to be effective.

There are concerns of new or worsening extra-articular features, including inflammatory eye disease in patients. In the etanercept trial for AS patients, several cases of uveitis/iritis (some with prior history, others with new diagnosis) as well as de novo colitis and psoriasis were reported [25]. In the study comparing adalimumab, infliximab, and etanercept in the treatment of uveitis in SpA patients, etanercept did not decrease the rate of uveitis flares in patients with at least one prior flare; the monoclonal anti-TNF antibodies did decrease the rate of uveitis flares [28]. There were five cases of new uveitis documented in the certolizumab study in AS patients [29]. However, there is a case of successful treatment of refractory SpA in a patient with uveitis with certolizumab [30].

Monoclonal antibodies to TNF- $\alpha$ and pegylated Fab' portion are approved for treatment of one or both inflammatory bowel diseases. Etanercept, however, failed to show efficacy in Crohn's disease [31].

\section{IL-17 Axis and IL-17 and IL-12/23 Inhibitors}

Over the last decade, our knowledge about the role of the Th-17/IL-23 axis in the pathogenesis of various inflammatory and immunological diseases has grown exponentially. Interleukin-17 (IL-17) is a notable cytokine of Th17 cells and mediates immune responses against bacterial and fungal infections, as well as in the pathogenesis of inflammatory and autoimmune diseases [32 $\bullet$. IL-17 is produced not only by Th17 cells but also by other cells of the innate immune system including dendritic cells, mast cells, macrophages, and natural killer (NK) cells [33•]. There is ample evidence that shows that IL-17 is highly upregulated at sites of inflamed tissues of inflammatory and autoimmune diseases and that it amplifies the inflammation through synergy with other cytokines, such as TNF- $\alpha$ [34]. IL-17A-producing mast cells and neutrophils are demonstrated in abundance in psoriatic plaque compared to normal skin, as well as in inflamed synovial tissue in AS patients [33•].

More recently, interleukin-12 (IL-12) and interleukin-23 (IL-23), cytokines that help drive an adaptive immune response by inducing naïve $\mathrm{CD} 4+$ lymphocytes to differentiate into Th1 cells and Th17 cells, have been identified as key mediators of inflammation in PsA and AS [35]. Novel approaches in the treatment of PsA and SpA involve blockade of the IL-17 axis by using a new class of biologics.

Ustekinumab is a monoclonal antibody that binds to the common $\mathrm{p} 40$

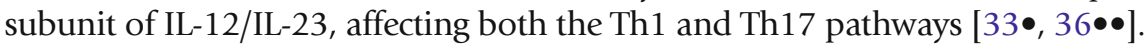
It is approved for the treatment of adult patients with moderate to severe plaque psoriasis, and for active PsA, alone or in combination with methotrexate. Efficacy and safety of ustekinumab compared to placebo in 186 patients with PsA who had spondylitis and peripheral joint involvement have also been recently reported in an abstract [37]. Data from 108 weeks trial showed that the proportion of patients with AEs were comparable between the placebo and combined ustekinumab-treated groups (AEs 32.9 vs $24.1 \%$; serious adverse events (SAEs) 1.4 vs $0.9 \%$; discontinuations due to AEs 2.9 vs $0.9 \%$; serious 
infections 14.3 vs $7.8 \%$ ). Through 2 years, safety observations were consistent with the overall PsA population.

More direct inhibitors, secukinumab and ixekizumab, are both monoclonal antibodies directed against IL-17A. Secukinumab has just been approved by FDA for the treatment of moderate to severe plaque psoriasis in adult patients, and the recently reported phase III studies show its effectiveness in PsA as well $[38,39]$. Most importantly, it provided a significant and sustained inhibition of joint structural damage in active psoriatic arthritis, regardless of prior TNF inhibitors or concomitant methotrexate [39].

Brodalumab is a human anti-IL-17 receptor A (IL-17RA) monoclonal antibody. Data from an open-label extension of a phase 2 study in adult patients with active PsA suggest that brodalumab is also a promising new biologic for patients with PsA [40]. Adverse effects were similar in brodalumab and placebo groups. These innovative treatment options have demonstrated improved clinical outcomes in both diseases. Although phase III trials are underway for these novel therapeutics in AS and PsA, some conclusions might be drawn from initial trials and bring light to comparing adverse effects to those of tumor necrosis factor inhibitors.

As IL-17 cytokine functions as part of the Th17 defense against extracellular pathogens, it is foreseeable to expect some increased risk of infections in patients on IL-17 blocker therapy. In the ERASURE (Efficacy of Response and Safety of Two Fixed Secukinumab Regimens in Psoriasis) trial, there were higher proportions of patients with infections and infestations in the secukinumab groups $(29.4 \%$ in the 300-mg group and $26.9 \%$ in the $150-\mathrm{mg}$ group) than in the placebo group

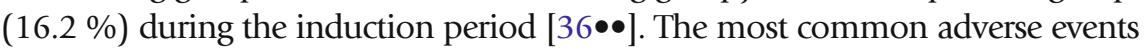
in the induction period and the entire treatment period in this study were nasopharyngitis, headache, and upper respiratory tract infection.

In pooled long-term safety data $[41,42]$ from the randomised, double-blind, placebo-controlled trial of ustekinumab in psoriasis patients [43, 44], which included patients on ustekinumab for as long as 3 years, rates of adverse events were low and comparable among all groups (1.2 to $1.9 \%$ ). No dose response in rates of AEs, overall infections, or SAEs was apparent through 3 years. Serious infections were listed as the most common serious adverse event with ustekinumab, a rate of 0.6 events per 100 patient-years in the 45-mg-dose group, and a rate of 1.4 events per 100 patient-years in the 90-mg-dose group. Rates of AEs, infections, SAEs, and AEs leading to study agent discontinuation remained generally stable or decreased over time. Pooled data from four clinical trials of ustekinumab in psoriasis patients were reviewed including 9000 patient-years of follow-up in 3117 patients treated for up to 5 years [45•]. Most observed AEs were non-serious and did not result in treatment discontinuation, and no clear signals of dose response or effects of cumulative exposure on safety outcomes were observed. These results confirm that the long-term safety profile of ustekinumab continues to be favorable and consistent with previous reports at 3 years follow-up [45•]. The difference initially observed in the incidence of serious infections between the 45- and 90-mg groups after up to 3 years of treatment continued to narrow with two additional years of follow-up, suggesting little difference in infection risk between the two doses. This infection rate was not elevated when compared to psoriasis patients on standard agents [42]. 
Similarly, during the brodalumab trial in PsA, the most frequent adverse events reported were respiratory infections (nasopharyngitis, upper respiratory tract infection (URTI), bronchitis, sinusitis) [46, 47]. During the blinded phase, the URTI rate was reported in $12 \%$ of brodalumab patients compared to $7 \%$ of placebo patients [46]. Likewise, in the secukinumab trial in AS, respiratory infection was one of the most common AE reported ( $29 \%$ in study patients); the incidence of infections was higher in the secukinumab group compared to the placebo [48].

\section{Tuberculosis and Opportunistic Infections}

At this time, both short- and long-term trials have not reported any tuberculosis or opportunistic infections in the IL-17 axis inhibitors. No reactivation of latent tuberculosis or viral hepatitis was observed in any psoriasis trial with ustekinumab [49].

Across five clinical trials of ustekinumab-treated patients with psoriasis, there were no atypical mycobacterial diseases, disseminated salmonellosis, or systemic fungal infections observed in ustekinumab-treated patients. In addition, no opportunistic infections were reported, except two previously reported cases of severe cutaneous herpes zoster infection with no evidence of visceral involvement [50]. The association of active tuberculosis and latent tuberculosis infection reactivation with some anti-TNF agents was not observed in these analyses. The possible pathophysiological explanation for this observation may be that during long-term use of ustekinumab treatment, IL-12/23 inhibition is possibly incomplete and does not compromise host defense towards these pathogens.

Also, no cases of LTBI reactivation were observed in patients receiving concomitant INH prophylaxis for LTBI. INH prophylaxis was generally well tolerated by these patients with psoriasis [51].

\section{Major Adverse Cardiovascular Events}

The second most frequent serious $\mathrm{AE}$ in the pooled ustekinumab data in psoriasis patient trials was cardiac disease; the rate was 0.8 events per 100 patient-years in the 45-mg-dose group and 1.3 events per 100 patient-years in the 90-mg-dose group [41, 42]. In a systematic review and meta-analysis in psoriatic patients who had received IL-12/23 inhibitors (ustekinumab or briakinumab), a higher risk of major adverse cardiovascular events (MACE) [including cardiovascular death, myocardial infarction, or stroke] was noted compared to those with placebo: OR 4.23 (95\% CI 1.07-16.75, $p=0.04$ ) [52]. However, this risk has not been seen with secukinumab when compared with placebo or etanercept. MACE have been reported for a similar proportion of patients on secukinumab and etanercept: $0.4 \%$ with $300 \mathrm{mg}, 0.4 \%$ with $150 \mathrm{mg}$, and $0.3 \%$ for etanercept.

A handful of solid and skin cancers have been reported in the short time the IL-17 axis inhibitors have been studied; non-melanomatous skin cancers (NMSCs) were the most commonly observed malignancy. In the pooled ustekinumab data in psoriasis patients, non-melanomatous malignancies occurred at a rate of 0.7 events per 100 patient-years in the 45-mg-dose group and a rate of 0.5 events per 100 patient-years in the 90-mg-dose group [41, 42]. At 3 years follow-up, this malignancy rate was similar to that in the general American population; the 
standardized incidence ratio was 1.05 (95 \% CI 0.69-1.53) [42]. In pooled psoriasis data from the PHOENIX 2 trial, over 5 years of follow-up, 0.66 malignancies (excluding NMSCs) were reported per 100 subject years of follow-up; 0.28 non-melanomatous skin cancers per 100 subject years of follow-up were reported [53].

Across the clinical trials of ustekinumab in psoriasis, the spectrum of malignancies observed was consistent with that expected in the general population. The incidence of malignancies other than NMSCs in ustekinumab-treated patients exposed up to 3 years was 0.60/100 patient-years [49]. This is consistent with that expected in the general US population [42]. Overall, the incidence of NMSCs in ustekinumab-treated patients is 0.52/100 patient-years [49].

The breast and skin cancers noted in the ustekinumab trial [54 $\bullet$ were found in patients with prior TNF-inhibitor exposure; similarly, there is one report of a basal cell cancer in a patient receiving ustekinumab for PsA, though it was unclear if this patient had received TNF-I previously [55]. It was not clear in the brodalumab trials whether the metastatic lung, breast, and skin cancer cases (one each) had received previous TNF-inhibitor treatment [46, 47].

Several reports of cytopenias have been noted in the IL-17 axis blocker trials. In the brodalumab study in PsA patients, there were four reports of grade 1 neutropenia (one in the 140-mg group, three in the 280-mg group) seen during the first 12 weeks, but none seen in the open-label 9-month extension of the trial [46]. In the secukinumab trial in PsA, grade 1 neutropenia was observed in five secukinumab patients and grade 2 in one patient [56•]. Similarly, several cases of grade 1 leukopenia and neutropenia were reported in AS patients receiving secukinumab, but the exact number of cases was not noted [48]. Recent studies have established an important role of Th17 cells, the manufacturers of IL-17, in the pathogenesis of acute myeloid leukemia $[57,58]$; it is possible that when a mechanism along these lines is blocked, such cytopenias are resultant.

Injection site erythema or injection site reactions have been noted in small numbers in both brodalumab and ustekinumab studies $(2-4 \%[46,47]$ and $0-$ $4 \%[55,59]$, respectively). New inflammatory arthritis was noted in two patients in a study of ustekinumab in psoriasis patients [60].

Secukinumab pooled data from the psoriasis trials has shown a very low risk of incident inflammatory bowel disease as well as exacerbation of prevalent inflammatory bowel disease. When compared to the etanercept-exposed psoriatic patients in the same pooled analysis, there was similar exposure-adjusted incidence of inflammatory bowel disease $(0.35 / 100$ patient-year incidence rate (IR) (95\% CI 0.10-0.90) for all exposed to the 150-mg dose; 0.26 IR (95\% CI 0.05-0.75) for the 300-mg dose; 0.34 IR (95\% CI 0.01-1.90) for the etanercept comparison group). These low rates are results of three reported cases of Crohn's, two existing cases with exacerbations, and one incident case with baseline symptoms suggesting possible undiagnosed inflammatory bowel disease. However, the FDA label for secukinumab cautions use in patients with 
known inflammatory bowel disease as well as monitoring for potential new bowel disease symptoms in other patients [61]. In the brodalumab trial, the presence of IBD was an exclusion criterion for entry to the study [46].

Head-to-Head Safety Comparison Between TNF-a Inhibitors and IL-17 Axis Inhibitors

The ACCEPT trial is a short clinical trial that provided comparative safety between ustekinumab to etanercept in the treatment of patients with plaque

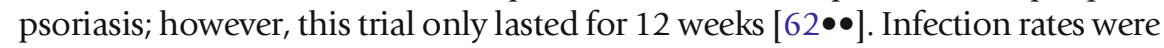
similar between the ustekinumab (45- and 90-mg doses) and etanercept groups, occurring in $29.1,30.6$, and $29.7 \%$ of patients. Adverse events of any kind were experienced by 70.0, 66.0, and $69.2 \%$ of patients. Mild injection site reactions were more common in the etanercept group-24.8 versus 4.3 and $3.7 \%$ in the ustekinumab group.

Another randomized, phase 3 trial, FIXTURE (Full Year Investigative Examination of Secukinumab vs. Etanercept Using Two Dosing Regimens to Determine Efficacy in Psoriasis), assessed the efficacy and safety of secukinumab, at a dose of 300 or $150 \mathrm{mg}$, compared to etanercept in patients with plaque psoriasis [36••]. The incidences of adverse events between the secukinumab and etanercept groups in the FIXTURE study during the entire 52-week treatment period were similar. The rates of serious adverse events were 6.8 events per 100 patient-years in the 300-mg secukinumab group, 6.0 events per 100 patient-years in the 150-mg secukinumab group, 7.0 events per 100 patient-years in the etanercept group, and 8.3 events per 100 patient-years in the placebo group. Infection rates were $26.7 \%$ with the 300 mg dose of secukinumab, $30.9 \%$ with the 150-mg dose of secukinumab, $24.5 \%$ with etanercept, and $19.3 \%$ with the placebo. Candida infections were more common with secukinumab (4.7 and $2.3 \%$ among the two doses) than with etanercept during the entire treatment period.

The incidence of injection site reactions during the entire study was lower in the combined secukinumab groups than in the etanercept group (7 patients [0.7\%] vs. 36 patients [11.1\%]).

At present, there are no comparative safety data between TNF- $\alpha$ inhibitors and IL-17 axis inhibitors except for two short-term clinical trials. TNF- $\alpha$ inhibitors have an advantage because of a well-defined safety profile based on more than a decade of use. However, based on the review of data from clinical trials, IL-17 axis inhibitors are equally safe. Rates of infection are comparable between the two groups, but risk of opportunistic infection particularly reactivation of TB is higher with TNF- $\alpha$ inhibitors.

Risk of malignancies with long-term use of biologics is still unclear. Based on observational data, malignancy risk other than NMSK has not been shown to be increased with the use of TNF- $\alpha$ inhibitors. With the short duration of availability, it is unclear if IL-17 axis inhibitors will reveal this same association. Based on current clinical trial data, risk of malignancy with IL-17 inhibitors is comparable to TNF- $\alpha$ inhibitors.

Higher rates of cardiovascular events have been noted in ustekinumab clinical trial data. However, increased rates of $\mathrm{CV}$ events have not been seen with secukinumab when used in clinical trials. Similarly, no increased CV events 
are seen with the TNF antagonist both from clinical trial and long-term registry data.

New-onset or worsening psoriatic plaques, particularly the plantar-palmarpustular type, have been reported with exposure to TNF inhibitors. This has not been seen with IL-17 or IL-12/23 inhibitors during the clinical trials, but there are few reports of pustular psoriasis with ustekinumab from post-marketing data. Both TNF- $\alpha$ inhibitors and IL-17 axis blockade agents have been noted to cause injection site erythema or local reactions; TNF- $\alpha$ inhibitors exhibited significantly more reactions than IL-17 axis inhibitors.

Uveitis usually improves with TNF blockers but paradoxically can worsen with the TNF-receptor blocker. At this time, there is no data noting the worsening of uveitis with any IL-17 axis blocker. There is a low risk of incident inflammatory bowel disease as well as exacerbation of prevalent inflammatory bowel disease with the anti-IL-17A monoclonal antibody. This has not been seen with TNF- $\alpha$ inhibitors except with etanercept.

In conclusion, there are very few discrepancies in adverse events of TNF blockade and IL-17 axis blockade therapies. Time-dependent differences in adverse events between the two classes of biologics may become evident. With longer-term and head-to-head studies, more clarity in understanding of the differential adverse effects may become obvious. However, one always needs to keep in mind the potential for complication like infections, malignancy, and cardiovascular and hematologic side effects when prescribing biologics.

\section{Compliance with Ethics Guidelines}

\section{Conflict of Interest}

Maria Antonelli declares that she has no conflict of interest.

Muhammad A. Khan has received consultancy/speaker fees from Abbvie, Amgen, Novartis, Celgene, and Crescendo Bioscience.

Marina N. Magrey has received clinical trial fees from Abbvie and UCB Pharma.

\section{Human and Animal Rights and Informed Consent}

This article does not contain any studies with human or animal subjects performed by any of the authors.

\section{References}

Papers of particular interest, published recently, have been highlighted as:

- Of importance

$\bullet \quad$ Of major importance

1. Khan MA. Update on spondyloarthropathies. Ann Intern Med. 2002;136(12):896-907.

2. Gladman DD, Antoni C, Mease P, et al. Psoriatic arthritis: epidemiology, clinical features, course, and outcome. Ann Rheum Dis. 2005;64 Suppl 2:ii14-7.
3. Davis JC, Mease PJ. Insights into the pathology and treatment of spondyloarthritis: from the bench to the clinic. Semin Arthritis Rheum. 2008;38(2):83-100.

4. Semble AL, Davis SA, Feldman SR. Safety and tolerability of tumor necrosis factor-a inhibitors in psoriasis: a narrative review. Am J Clin Dermatol. 2014;15:37-43. 
This very thorough narrative review discusses safety and tolerability of TNF-inhibitors in the treatment of moderate-to-severe plaque psoriasis.

5. Bradley JR. TNF-mediated inflammatory disease. J Pathol. 2008;214:149-60.

6. Aggarwal BB, Gupta SC, Kim JH. Historical perspectives on tumor necrosis factor and its superfamily: 25 years later, a golden journey. Blood. 2012;119(3):651-65.

7.• Dommasch ED, Abuabara K, Shin DB, Nguyen J, Troxel $\mathrm{AB}$, Gelfand JM. The risk of infection and malignancy with tumor necrois factor antagonists in adult patients with psoriatic disease: a systematic review and metaanalysis of randomized controlled trials. J Am Acad Dermatol. 2011;64(6):1035-50.

This excellent systematic review and meta-analysis reviews the risks of infection and malignancy in TNF-blocker use in patients with PsA.

8. Mease PJ, Ory P, Sharp JT, Ritchlin CT, Van den Bosch F, Wellborne F, et al. Adalimumab for long-term treatment of psoriatic arthritis: 2-year data from the Adalimumab Effectiveness in Psoriatic Arthritis Trial (ADEPT). Ann Rheum Dis. 2009;68:702-9.

9. Van der Heijde DM, Schiff MH, Seiper J, Kivitz AJ, Wong RL, Kupper H, et al. Adalimumab effectiveness for the treatment of ankylosing spondylitis is maintained for up to 2 years: long-term results from the ATLAS trial. Ann Rheum Dis. 2009;68:922-9.

10. Atzeni F, Sarzi-Puttini P, Botsios C, Carletto A, Cipriani $\mathrm{P}$, Gavalli EG, et al. Long-term anti-TNF therapy and risk of serious infections in a cohort of patients with rheumatoid arthritis: comparison of adalimumab, etanercept and infliximab in the GISEA registry. Autoimmun Rev. 2012;12:225-9.

11. Listing J, Strangfeld A, Kary S, et al. Infections in patients with rheumatoid arthritis treated with biologic agents. Arthritis Rheum. 2005;52:3403-12.

12. Keane J. TNF-blocking agents and tuberculosis: new drugs illuminate an old topic. Rheumatology. 2005;44:714-20.

13. Wallis RS, Broder M, Wong J, Beenhouwer D. Granulomatous infection due to tumor necrosis factor blockade: correction. Clin Infect Dis. 2004;39(15):1255-6.

14. Tubach F, Salmon D, Ravaud P, Allanore Y, Gaoupille $\mathrm{P}$, Breban M, et al. Risk of tuberculosis is higher with anti-tumor necrosis factor monoclonal antibody therapy than with soluble tumor necrosis factor receptor therapy: the three-year prospective French Research Axed on Tolerance of Biotherapies registry. Arthritis Rheum. 2009;60(7):1884-94.

15. Dixon WG, Hyrich KL, Watson KD, Lunt M, Galloway J, Ustianowski A, et al. Drug-specific risk of tuberculosis in patients with rheumatoid arthritis treated with antiTNF therapy: results from the British Society for Rheumatology Biologics Register (BSRBR). Arthritis Rheum Dis. 2010;69(3):522-8.

16. Salmon-Ceron D, Tubach F, Lortholary O, Chosidow $\mathrm{O}$, Bretagne S, Nicolas N, et al. Drug-specific risk of non-tuberculosis opportunistic infections in patients receiving anti-TNF therapy reported to the 3 -year prospective French RATIO registry. Ann Rheum Dis. 2011;70(4):616-23.

17. Serac G, Tubach F, Mariette X, Salmon-Ceron D, Ravaud $\mathrm{P}$, Liote $\mathrm{F}$, et al. Risk of herpes zoster in patients receiving anti-TNF- $\alpha$ in the prospective French RATIO Registry. J Invest Dermatol. 2012;132:726-9.

18. Lanternier F, Tubach F, Ravaud P, Salmon D, Dellamonica P, Bretagne S, et al. Incidence and risk factors of Legionella pneumophila pneumonia during anti-tumor necrosis factor therapy. Chest. 2013;144(3):990-8.

19.• Burmester GR, Panaccione R, Gordon KB, McIlraith MJ, Lacerda APM. Adalimumab: longterm safety in 23458 patients from global clinical trials in rheumatoid arthritis, juvenile idiopathic arthritis, ankylosing spondylitis, psoriatic arthritis, psoriasis and Crohn's disease. Ann Rheum Dis. 2013;72:517-24.

This large analysis includes evaluation of 71 trials world-wide including patients from plethora autoimmune diseases for nearly 12 years of adalimumab exposure. The analysis indicated a safety profile similar to prior anti-TNF studies.

20. Mariette X, Tubach F, Bagheri H, Bardet $M$, Berthelot JM, Gaudin P, et al. Lymphoma in patients treated with anti-TNF: results of the 3-year prospective French RATIO registry. Ann Rheum Dis. 2009;69(2):400-8.

21. Westhovens I, Lories RJ, Westhovens R, Verchueren P, de Vlam K. Anti-TNF therapy and malignancy in spondyloarthritis in Leuven spondyloarthritis biologics cohort (BIOSPAR). Clin Exp Rheumatol.

2014;32(1):71-6.

22. Mease PJ, Fleischmann R, Deodhar AA, Wollenhaupt J, Khraishi M, Kielar D, et al. Effect of certolizumab pegol on signs and symptoms in patients with psoriatic arthritis: 24-week results of a phase 3 double-blind randomized placebo-controlled study (RAPID-PsA). Ann Rheum Dis. 2014;73(48).

23. Inman RD, Davis JC, van der Heijde D, Diekman L, Sieper J, Kim SI, et al. Efficacy and safety of golimumab in patients with ankylosing spondylitis. Arthritis Rheum. 2008;58(11):3402-12.

24. Kavanaugh A, McInnes I, Mease P, Krueger GG, Gladman D, Gomez-Reino J, et al. Golimumab, a new human tumor necrosis factor alpha antibody, administered every four weeks as a subcutaneous injection in psoriatic arthritis. Arthritis Rheum. 2009;60(4):976-86.

25. Davis J, der Heijde DM V, Braun J, Dougados M, Cush J, Clegg D, et al. Sustained durability and tolerability of etanercept in ankylosing spondylitis for 96 weeks. Ann Rheum Dis. 2005;64(11):1557-62.

26. Hastings R, Ding T, Butt S, Gadsby K, Zhang W, Moots $\mathrm{RJ}$, et al. Neutropenia in patients receiving anti-tumor necrosis factor therapy. Arthritis Care Res. 2010;62(6):764-9. 
27. U.S. Food and Drug Administration. Center for Drug Evaluation and Research. Safety information for tumor necrosis factor (TNF) blockers. http://www.fda.gov/Drugs/ DrugSafety/PostmarketDrugSafetyInformationforPatients andProviders/DrugSafetyInformationforHeathcare Professionals/ucm174474.htm Accessed February 16, 2015.

28. Guignard S, Gossec L, Salliot C, RuyssenWitrand A, Luc M, Duclos M, et al. Efficacy of tumor necrosis factor blockers in reducing uveitis flares in patients with spondyloarthropathy: a retrospective study. Ann Rheum Dis. 2006;65:1631-4.

29. Landewe R, Braun J, Deodhar A, et al. Efficacy of certolizumab pegol on signs and symptoms of axial spondyloarthritis including ankylosing spondylitis: 24week results of a double-blind randomized placebocontrolled phase 3 study. Ann Rheum Dis. 2014;73:39-47.

30. Maiz Alonso O, Blaco Esteban AC, Eques Dubuc CA, Martinez Zabalequi D. Effectiveness of certolizumab pegol in chronic anterior uveitis associated to Crohn's disease and ankylosing spondylitis. Rheumatol Clin. 2014; epub ahead of print.

31. Sandborn WJ, Hanauer SB, Katz S, Safdi M, Wolf DG, Baerg RD, et al. Etanercept for active Crohn's disease: a randomized, double-blind, placebocontrolled trial. Gastroenterology.

2001;121(5):1088-94.

32. Zhu S, Qian Y. IL-17/IL-17 receptor system in autoimmune disease: mechanisms and therapeutic potential. Clin Sci (Lond). 2012;122(11):487-511.

This review discusses the IL-17 axis role against bacterial and fungal infections in addition to its role in pathogenesis of autoimmune disease.

33. Dhavalkumar DP, Lee DM, Kolbinger F, Antoni C. Effect of IL-17A blockade with secukinumab in autoimmune diseases. Ann Rheum Dis. 2013;72:ii116-23.

This excellent review article discusses the role of IL-17a in immunity and autoimmune disease as well as using IL-17 as a target for therapeutic interventions.

34. Frelata M, Siebert S, McInnes IB. The interleukin-17 pathway in psoriasis and psoriatic arthritis: disease pathogenesis and possibilities of treatment. Curr Rheumatol Rep. 2014;16:414.

35. McKenzie BS, Kastelein RA, Cua DJ. Understanding the IL-23-IL-17 immune pathway. Trends Immunol. 2006;27:17-23.

36.• Langley RG, Elewski BE, Lebwohl M, Reich K, Gfittiths CE, Papp K, et al. Secukinumab in plaque psoriasis-results of two phase 3 trials. New Engl J Med. 2014;371:326-38.

This excellent paper describes two phase 3, double-blind, yearlong psoriasis trials ERASURE and FIXTURE comparing secukinumab at two different doses to placebo, and secukinumab to etanercept, respectively. The results indicate secukinumab is effective in psoriasis and the safety profile indicates a higher infection rate of secukinumab compared to placebo, but comparable to etanercept.
37. Kavanaugh A, Sanz LP, Gottlieb AB, et al. Efficacy and safety of ustekinumab in psoriatic arthritis patients with spondylitis and peripheral joint involvement: results from a phase 3, multicenter, double-blind placebo-controlled study. Arthritis Rheum.

2014;66(suppl1):539. Abstract.

38. McInnes IB, Mease PJ, Kikham B, et al. Secukimumab, a human anti-interleukin-17A monoclonal antibody, improves psoriatic arthritis: 24 week efficacy and safety data from a phase 3 randomized multicenter, double-blind, placebo-controlled study using subcutaneous dosing. ACR 2014, Arthritis Rheum. 2014;66(suppl1):L1. Abstract.

39. van der Heijde D, Landewe RBM, Mease P, et al. Secukimumab, a monoclonal antibody to interleukin$17 \mathrm{~A}$, provides significant and sustained inhibition of joint structural damage in active psoriatic arthritis, regardless of prior TNF inhibitors or concomitant methotrexate: a phase 3 randomized, double-blind, placebo-controlled study. ACR 2014, Arthritis Rheum. 2014;66(suppl1):954. Abstract.

40. Genovese MC, Mease PJ, Greenwald MW, et al. Clinical response in subjects with psoriatic arthritis following one year of treatment with brodalumab, an anti-IL-17 receptor antibody. ACR 2014, Arthritis Rheum. 2014;66(suppl1):1557. Abstract.

41. Lebwohl M, Leonardi G, Griffiths CEM, et al. Longterm safety experience of ustekinumab in patients with moderate-to-severe psoriasis (part I of II): results from analysis of general safety parameters from pooled phase 2 and 3 clinical trials. J Am Acad Dermatol. 2012;66(5):731-41.

42. Gordon KB, Pap KA, Langley RG, et al. Long-term safety experience of ustekinumab in patients with moderate to severe psoriasis (part II of II): results from analysis of infections and malignancy from pooled phase II and III clinical trials. J Am Acad Dermatol. 2012;66(5):74251.

43. Leonardi CL, Kimball AB, Papp KA, et al. Efficacy and safety of ustekinumab, a human interleukin-12/23 monoclonal antibody in patients with psoriasis: $76-$ week results from a randomized, double-blind, placebo controlled trial (PHOENIX 1). Lancet. 2008;371:1665-74.

44. Papp KA, Langley RG, Lebwohl M, et al. Efficacy and safety of ustekinumab, a human interleukin-12/23 monoclonal antibody, in patients with psoriasis: $52-$ week results from a randomized, double blind, placebo- controlled trial (PHOENIX 2). Lancet. 2008;371:1675-84.

45. Papp KA, Griffiths CE, Gordon K, Lebwohl M, et al. Long-term safety of ustekinumab in patients with moderate-to-severe psoriasis: final results from 5 years of follow-up. Br J Dermatol. 2013;168:84454.

This study demonstrated the ustekinumab safety profile in psoriasis patients treated up to 5 years. The study showed no dose-related or cumulative toxicity with an acceptable safety profile. 
46. Mease PJ, Genovese MC, Greenwald MW, Ritchlin CT, Beaulieu A, Deodhar AA, et al. Brodalumab, an antiIL17RA monoclonal antibody, in psoriatic arthritis. New Engl J Med. 2014;370:2295-306.

47. Genovese MC, Mease PJ, Greenwald MW, Ritchlin CT, Beaulieu A, Deodhar AA, et al. Clinical response in subjects with psoriatic arthritis following one year of treatment with brodalumab, an anti-interleukin-17 receptor antibody. ACR 2014 abstract \#1557. Arthritis Rheum. 2014;66:11-S687.

48. Baeten D, Baraliakos X, Braun J, Sieper J, Emery P, van der Heijde D, et al. Anti-interleukin-17A monoclonal antibody secukinumab in treatment of ankylosing spondylitis: a randomized, double-blind, placebocontrolled trial. Lancet. 2013;382:1705-13.

49. U.S. Food and Drug Administration. Center for Drug Evaluation and Research. Safety information for ustekinumab. http://www.fda.gov/Safety/MedWatch/ SafetyInformation/ucm311305.htm Accessed January 20, 2015.

50. Failla V, Nikkels AF. Ustekinumab and herpes zoster. Dermatology. 2011;222(2):119-22.

51. Tsai TF, Ho V, Song M, Szapary P, et al. The safety of ustekinumab treatment in patients with moderate-tosevere psoriasis and latent tuberculosis infection. $\mathrm{Br} \mathrm{J}$ Dermatol. 2012;167:1145-52.

52. Tzellos T, Kyrgidis A, Zouboulis CC. Re-evaluation of the risk of major cardiovascular events in patients treated with anti-IL-12/23 biological agents for chronic plaque psoriasis: a meta-analysis of randomized controlled trials. Eur Acad Dermatol Venereol.

2013;27(5):622-7.

53. Langley RG, Lebwohl M, Krueger GG, Szapary PO, Wasfi Y, Chan D, Hsu, et al. Long-term efficacy and safety of ustekinumab with and without dosing adjustment in patients with moderate-to-severe psoriasis: results from the PHOENIX 2 Study through 5 years of follow up. Br J Dermatol. 2014; epub ahead of print.

54. Ritchlin C, Rahman P, Kavanaugh A, McInnes IB, Puig L, Li S, et al. Efficacy and safety of the anti-IL-12/23 p40 monoclonal antibody, ustekinumab, in patients with active psoriatic arthritis despite conventional non-biological and biological anti-tumor necrosis factor therapy: 6-month and 1-year results of the phase 3, multicenter, double-blind, placebo-controlled, randomized PSUMMIT 2 trial. Ann Rheum Dis. 2014;73:990-9.

This six-month trial demonstrated significant ustekinumab efficacy in PsA patients without unexpected AE through week 60.

55. Gottlieb A, Menter A, Mendelsohn A, Shen YK, Li S, Guzzo C, et al. Ustekinumab, a human interleukin 12/ 23 monoclonal antibody for psoriatic arthritis: randomized, double-blind, placebo-controlled, crossover trial. Lancet. 2009;373:633-40.

56. McInnes IB, Sieper J, Braun J, Emery P, van der Heijde D, Isaacs JD, et al. Efficacy and safety of secukinumab, a fully human anti-interleukin-17A monodonal antibody, in patients with moderate-to-severe psoriatic arthritis: a 24week, randomized, double-blind, placebo-controlled, phase II proof-of-concept trial. Ann Rheum Dis. 2014;73:349-56.

This trial's intent was to investigate the safety and efficacy of secukinumab in PsA.The AE profile was noted to be comparable between secukinumab and placebo.

57. Han Y, Ye A, Bi L, Wu J, Yu K, Zhang S. Th17 cells and interleukin-17 increase with poor prognosis in patients with acute myeloid leukemia. Cancer Sci.

2014;105(8):933-42.

58. Wu C, Wang S, Wang F, Chen Q, Peng S, Zhang Y, et al. Increased frequencies of $\mathrm{T}$ helper type 17 cells in the peripheral blood patients with acute myeloid leukaemia. Clin Exp Immunol. 2009;158(2):199-204.

59. Poddubnyy D, Hermann KGA, Callhoff J, Listing J, Siper J. Ustekinumab for the treatment of patients with active ankylosing spondylitis: results of a 28 -week, prospective, open-label, proof-of-concept study (TOPAS). Ann Rheum Dis. 2014;73:817-23.

60. De Souza A, Ali-Shaw T, Redy SM, Fiorentino D, Strober BE. Inflammatory arthritis following ustekinumab treatment for psoriasis: a report of two cases. Br J Dermatol. 2013;168(1):210-12.

61. U.S. Food and Drug Administration. Center for Drug Evaluation and Research. Safety information for secukinumab. http://www.fda.gov/downloads/ AdvisoryCommittees/CommitteesMeetingMaterials/ Drugs/DermatologicandOphthalmicDrugsAdvisory Committee/UCM419023.pdf Accessed February 6, 2015.

62.• Griffiths EM, Strober BE, van de Kerkhof P, et al. Comparison of ustekinumab and etanercept for moderate-tosevere psoriasis. N Eng J Med. 2010;362:118-28.

This randomized, non-blinded trial compared two doses of ustekinumab to etanercept in moderate-to-severe psoriasis patients. Both the $45 \mathrm{mg}$ and $90 \mathrm{mg}$ doses of ustekinumab were found to be superior to etanercept over the 12-week trial; the safety profile was similar for both medications.

63. Mease PJ, Goffe BS, Metz J, VanderStoep A, Finck B, Burge DJ. Etanercept in the treatment of psoriatic arthritis and psoriasis: a randomized trial. Lancet. 2000;356:385-90.

64. Antoni CE, Krueger GG, de Vlam K, Birbara C, Beutler A, Guzzo C, et al. Infliximab improves signs and symptoms of psoriatic arthritis: results of the IMPACT 2 trial. Ann Rheum Dis. 2005;64:1150-57.

65. Antoni CE, Kavanaugh A, Kirkham B, Tutuncu Z, Burmester GR, Schneider U, et al. Sustained benefits of infliximab therapy for dermatologic and articular manifestations of psoriatic arthritis. Arthritis Rheumatism. 2005;52(4):1227-36.

66. Braun J, Brandt J, Listing J, Zink A, Alten R, Burmester $\mathrm{G}$, et al. Two year maintenance of efficacy and safety of infliximab in the treatment of ankylosing spondylitis. Ann Rheum Dis. 2005;34:229-34.

67. Sieper J, Landewe R, Rudwaleit M, van der Heijde D, et al. Effect of certolizumab Pegol over 96 weeks in patients with axial spondyloarthritis: results from a phase 3 randomized trial. Arthritis Rheumatol. 2014;epub ahead of print. 